\title{
Ganancias de bienestar por nuevas variedades importadas. Evidencia para España, 1988-2006*
}

\author{
Asier Minondo \\ Deusto Business School \\ Francisco Requena-Silvente \\ Universitat de València
}

\section{Resumen}

Este trabajo investiga las ganancias de bienestar a través de las importaciones españolas de nuevas variedades entre 1988 y 2006 usando la metodología propuesta por Feenstra (1994) y Broda y Weinstein (2006). Después de calcular la elasticidad de sustitución para un gran número de productos importados, estimamos que la ganancia de bienestar asociada a la proliferación de variedades importadas en España es equivalente al 1,2 por 100 del PIB de 2006. A continuación descomponemos la contribución que tiene cada país exportador a dicha ganancia de bienestar. China aparece como el país más importante con una contribución cercana al 12 por 100, casi la misma que todo el conjunto de la UE-15.

Palabras claves: ganancias del comercio, comercio de variedades, importaciones españolas.

Clasificación JEL: F12, F14.

\section{Abstract}

This paper investigates the welfare gains due to Spanish imports of new varieties over the period 1988-2006 using the methodology proposed by Feenstra (1994) and Broda and Weinstein (2006). After calculating the elasticities of substitution of a large number of Spanish imported products, we estimate that the total welfare gain due to imports of new varieties in Spain is equal to 1.2 per cent of GDP in 2006. Next we decompose the contribution of each country to the total welfare gain. By countries, China accounts for about 12 per cent of the total gain, almost the same as the entire EU-15.

Keywords: gains from trade, trade in varieties, Spanish imports.

JEL classification: F12, F14.

\section{Introducción}

El acceso al mercado mundial de productos permite a los consumidores de un país acceder no solamente a productos más baratos sino también a nuevas variedades de esos productos hasta el momento desconocidas. Krugman $(1979,1980)$ fue el primero en formalizar en un modelo teórico la idea de «el amor por la variedad»

* Asier Minondo agradece la ayuda financiera del Departamento de Educación, Universidades e Investigación del Gobierno Vasco y del Ministerio de Educación y Ciencia (proyecto ECO 201021643). Francisco Requena agradece la ayuda financiera del Ministerio de Educación y Ciencia (proyecto ECO 2011-27619), así como de la Generalitat Valenciana (proyecto Prometeo/2009/098). 
como nueva fuente de ganancias de bienestar a través del comercio internacional. Veinticinco años después, Broda y Weinstein (2006) son los primeros en estimar empíricamente la magnitud de esos beneficios asociados al consumo de nuevas variedades importadas para un país. Los autores construyen un índice de precios de importación exacto agregado a partir del trabajo de Feenstra (1994), que elaboró un índice de precios de importación exacto para solamente un producto que tenía en cuenta el sesgo en los precios de importación por cambios en el número de variedades disponibles de ese producto. Dicho sesgo de importación mide cuánto estarían dispuestos a pagar los consumidores por acceder a un número mayor de variedades al final de un periodo determinado respecto al número de variedades disponibles al principio del periodo. Usando datos de importaciones de EEUU con un alto grado de desagregación de producto, los autores estiman que las nuevas variedades importadas reducen el índice de precios de importación convencional entre 1972 y 2001 en un 28 por 100 ( 1,2 por 100 cada año). Esto se traduce en una ganancia de bienestar acumulada durante este periodo del 2,6 por 100 del PIB de $2001^{1}$.

El número de trabajos empíricos que utilizan esta metodología todavía es escaso. Mohler (2009) y Mohler y Seitz (2010) calculan las ganancias de bienestar por consumo de variedades en Suiza entre 1990 y 2006 y en la UE-27 entre 1999 y 2008, respectivamente. Bloningen y Soderbery (2010) investigan las ganancias de bienestar por la importación de un producto diferenciado en un mercado concreto (el del automóvil en EEUU) usando datos de mercado en lugar de datos de aduanas. Los resultados apuntan a que las ganancias por proliferación de variedades son más del doble que las previamente estimadas cuando se utiliza una definición de producto y de variedad más precisa. Nuestro trabajo adopta la metodología de Broda y Weinstein con el fin de estimar las ganancias de bienestar por importación de nuevas variedades en el caso de España durante el periodo 1988-2006. A continuación, calculamos la importancia relativa de varias áreas geográficas y países concretos en los beneficios obtenidos a través del acceso a nuevas variedades importadas. El resto del trabajo está organizado de la siguiente manera. La sección 2 presenta de una manera sucinta los fundamentos teóricos y la estrategia empírica, dejando en el Apéndice las cuestiones técnicas para el lector interesado. La sección 3 presenta una descripción de los datos y la sección 4 comenta los resultados obtenidos. La sección 5 recoge las principales conclusiones del trabajo.

\footnotetext{
${ }^{1}$ Hay dos trabajos previos que han intentado calcular las ganancias de bienestar por acceso a nuevas variedades importadas. ROMER (1994) calibra un modelo de equilibrio general en el que el importador es una economía pequeña incapaz de producir sus propias variedades y muestra que la pérdida de bienestar por pérdida de variedades importadas puede alcanzar hasta el 20 por 100 del PIB cuando el país impone un arancel tan bajo como del 10 por 100. KLENOW y RODRIGUEZ-CLARE (1997) generaliza el modelo de Romer permitiendo que el país pueda producir sus propias variedades en cada industria, haciendo que el efecto negativo del arancel siga existiendo aunque su magnitud sea menor.
} 


\section{Fundamentos teóricos}

\subsection{Consideraciones preliminares}

En este trabajo cuantificamos los beneficios del crecimiento de las variedades importadas en un modelo de competencia monopolística. Para este fin es conveniente primero definir qué es una variedad. Para ello adoptamos el supuesto de Armington (1969), esto es, los productos comerciados internacionalmente son considerados como diferenciados en función del país de procedencia del producto. Así pues, una variedad es simplemente un producto producido en un país concreto. Desde el punto de vista empírico esto significa que el número de productos es constante de modo que la única fuente de ganancias es un cambio en el número de países exportadores (o variedades) de cada producto que un país importa.

A continuación necesitamos una forma de expresar cómo valoran las variedades los consumidores. La elección de una función de utilidad CES (con elasticidad de sustitución constante) es muy conveniente. Es fácil de tratar analíticamente y la estructura de demanda que se obtiene es simple, pero aun así permite incluir cambios en los precios agregados entre mercados. Para cada producto Feenstra (1994) demuestra que la forma funcional de la utilidad CES permite obtener un índice de precios exacto que incluye la entrada y salida de nuevos productos simplemente añadiendo un término adicional que ajusta el índice de precios convencional al tener en cuenta que los consumidores están dispuestos a pagar por acceder a nuevas variedades que perciben como diferentes entre sí para un producto concreto. Broda y Weinstein (2006) extienden este resultado de un producto a todos los productos importados por una economía obteniendo un índice de precios exacto agregado que tiene en cuenta el crecimiento del número de variedades a lo largo de un periodo de tiempo.

Por último, la función de utilidad CES y su índice de precios exacto correspondiente requieren conocer la elasticidad de sustitución entre variedades de un producto concreto. La elasticidad de sustitución nos informa sobre cómo de indiferentes son los consumidores con respecto al número de variedades disponibles. Desde un punto de vista empírico, estimar la elasticidad de sustitución a nivel de producto ofrece información relevante sobre el grado de sustitución entre variedades. Si la elasticidad de sustitución es alta para un producto concreto, entonces los consumidores tenderán a ser indiferentes entre las variedades disponibles; es decir, los consumidores no perciben como diferentes esas variedades simplemente por venir de distintos países, y por lo tanto las ganancias por nuevas variedades serán muy pequeñas. En el caso contrario, valores bajos de la elasticidad de sustitución indican que a los consumidores sí les importa que las variedades sean diferentes y, por tanto, las ganancias potenciales derivada de su importación son mayores. 


\subsection{Estrategia empírica}

A continuación describimos de forma sucinta nuestra estrategia empírica, invitando a la lectura de Feenstra (1994) y Broda y Weinstein (2006) para más detalles sobre esta metodología. El punto de partida es una función de utilidad tipo CES y el supuesto de Armington (1969) por el que una variedad es un producto g procedente del país c:

$$
M_{g r}=\left(\sum_{c \in C} d_{g c t}^{1 / \sigma_{g}}\left(m_{g c t}\right)^{\left(\sigma_{g}-1\right) / \sigma_{g}}\right)^{\sigma_{g} / \sigma_{g}-1}
$$

donde $\mathrm{C}$ es el conjunto de países (conjunto de posibles variedades) en el periodo t; $m_{g c t}$ es la sub-utilidad derivada del consumo de la variedad importada c del producto $\mathrm{g}$ en el periodo $\mathrm{t} ; d_{g c t}>0$ es el parámetro de preferencias correspondiente; y $\sigma>1$ es la elasticidad de substitución entre variedades. Sato (1976) deriva un índice de precios convencional para un producto, cuando el conjunto de variedades disponible, $I_{g}$, no cambia a lo largo del tiempo:

$$
P_{g}=\prod_{c \in I_{g}}\left(\frac{p_{g c t}}{p_{g c t-1}}\right)^{\omega_{g c t}}
$$

donde $\omega_{g c t}$ es el ponderador de variedad (ecuaciones A1 y A2 del Apéndice). Feenstra (1994) demuestra que el índice de precios exacto cuando se permite la entrada y salida de variedades a lo largo del tiempo es:

$$
\pi_{g}=P_{g}\left(\frac{\lambda_{g t}}{\lambda_{g t-1}}\right)^{1 /\left(\sigma_{g}-1\right)}
$$

donde

$$
\lambda_{g r}=\frac{\sum_{c \in C} p_{g c r} q_{g c r}}{\sum_{c \in I_{g r}} p_{g c r} q_{g c r}} \text { para } r=t, t-1
$$

El índice de precios exacto $\pi_{g}$ es el índice de precios convencional multiplicado por un término adicional, la ratio de lambdas, que mide la influencia del cambio en el número de variedades en los precios. El numerador de la ratio $\lambda_{g t}$ es el cociente entre el gasto en variedades disponibles en ambos periodos $\left(c \in I_{g}=\left(I_{g t} \cap I_{g t-1}\right)\right)$ y el gasto en variedades disponibles en el periodo $\mathrm{t}\left(c \in I_{g t}\right)$. Por lo tanto, a medida que aparezcan nuevas variedades el numerador $\lambda_{g t}$ disminuye y con ello la ratio de lambdas. El denominador de la ratio $\lambda_{g t-1}$ mide el impacto de la desaparición de variedades. Cuando más variedades desaparecen, menor es $\lambda_{g t-1}$ y por tanto mayor es la ratio de lambdas. La ratio de lambdas depende de la elasticidad de substitución: cuando la elasticidad de substitución es alta (como es el caso de bienes homogéneos), la ratio de lambdas tiende a 1 y su influencia en el índice de precios exacto tiende a ser pequeño. 
Broda y Weinstein (2006) construyen un índice de precios agregado exacto, donde aparece una ratio de lambdas que captura el impacto equivalente sobre precios de un cambio en el número de variedades de todos y cada uno de los productos, debidamente ponderado por el peso que tiene cada producto en las importaciones. Nuestro interés es calcular esa ratio entre el índice de precios agregado exacto y el índice de precios agregado convencional. Dicha ratio recibe el nombre de ratio de punto final (end-point ratio, EPR):

$$
E P R=\prod_{g \in G}\left(\frac{\pi_{g t}}{\pi_{g t}}\right)^{\omega_{g t}}=\prod_{g \in G}\left(\frac{\lambda_{g t}}{\lambda_{g t-1}}\right)^{\omega_{g t}\left(\sigma_{g}-1\right)}
$$

donde $\omega_{g t}$ es un factor de ponderación de producto (ecuaciones A3 y A4 del Apéndice). Ahora la ratio de punto final mide el sesgo en precios de una economía resultado de los cambios en las variedades de todos los productos a lo largo del tiempo: Si el EPR es inferior a 1, entonces el índice de precios convencional está sesgado al alza -es decir, el cambio de variedades debe de ajustar a la baja el índice de precios de importación convencional. Esta es la fuente de ganancia por nuevas variedades. Por último las ganancias de bienestar por el crecimiento de las variedades (GV) como porcentaje del PIB del país importador en el último año del periodose obtiene, a partir de ponderar la inversa de EPR por el peso total de las importaciones en el PIB como factor de ponderación:

$$
G V=\left[\prod_{g \in G}\left(\frac{\lambda_{g t}}{\lambda_{g t-1}}\right)^{-\omega_{g t} /\left(\sigma_{g}-1\right)}\right]^{\omega_{t}^{M}}
$$

donde $\omega_{g t}$ es un factor de ponderación de las importaciones (ecuaciones [A5] y [A6] del Apéndice).

\subsection{Método de estimación}

El proceso completo de cálculo de las ganancias de bienestar por el aumento de variedades importadas desde un país concreto se obtiene siguiendo estos pasos:

1. Definición del conjunto de productos $\mathrm{G}$ en el periodo seleccionado;

2. Cálculo de la elasticidad de substitución para cada producto, $\sigma_{g}$.

3. Cálculo de las lambdas $\lambda_{g t}$ y $\lambda_{g t-1}$ para cada producto.

4. Cálculo de la ratio de lambdas utilizando $\sigma_{g}, \lambda_{g t}$ y $\lambda_{g t-1}$, para conocer la diferencia entre el índice exacto de precios con cambios en variedades y el índice convencional de precios.

5. Cálculo de los ponderadores $\left(\omega_{g t}\right)$ asociados al cambio de precios decada producto con el fin de obtener una estimación del sesgo de importaciones en el índice agregado exacto de precios (EPR). 
6. Cálculo de las ganancias o pérdidas de bienestar en el cambio de precios utilizando el peso de las importaciones de bienes en el PIB durante el periodo (GV).

7. Bootstrap del proceso entero para obtener los errores estándar de $\sigma_{g}$, ratio de lambdas, EPR y GV. En este trabajo se han utilizado 50 repeticiones.

\subsection{Elasticidad de substitución}

Aquí explicamos cómo calcular la elasticidad de substitución de cada producto $\left(\sigma_{g}\right)$. Siguiendo a Feenstra (1994), existe una ecuación de demanda de importaciones para cada variedad de producto que puede expresarse en términos de cuotas y cambio en el tiempo como:

$$
\Delta \ln s_{g c t}=\varphi_{g t}-\left(\sigma_{g}-1\right) \Delta \ln p_{g c t}+\varepsilon_{g c t}
$$

donde $\varphi_{g t}$ es un efecto aleatorio específico por producto y año y $\varepsilon_{g c t}$ es otro término aleatorio derivado de las preferencias aleatorias de los consumidores hacia las distintas variedades.

Los productores compiten en variedades en mercados con estructura de competencia monopolísticade modo que los precios en primeras diferencias son:

$$
\Delta \ln p_{g c t}=\varphi_{g t}+\frac{\omega_{g}}{1+\omega_{g}} \Delta \ln s_{g c t}+\delta_{g c t}
$$

donde $\omega_{g}>0$ es la inversa de la elasticidad de oferta para un producto (idéntica para todas las variedades del mismo producto), $\psi_{g t}$ captura los shocks de producción específicos al par (producto-año) y $\delta_{g c t}$ captura los cambios tecnológicos en la producción de cada variedad.

Es obvio que las cuotas y los precios están endógenamente determinados: los shocks de demanda $\varepsilon_{g c t}$ o de oferta $\delta_{g c t}$ están ambos correlacionados con las cuotas y los precios. Para controlar este problema de endogeneidad estimamos estas ecuaciones simultáneamente usando la metodología propuesta por Feenstra (1994) y mejorada por Broda y Weinstein (2006). El primer paso es eliminar $\varphi_{g t}$ y $\psi_{g t}$ eligiendo un país de referencia $\mathrm{k}$ y diferenciando las ecuaciones de demanda y oferta, [6] y [7], respecto al país k:

$$
\begin{gathered}
\Delta^{k} \ln s_{g c t}=-\left(\sigma_{g}-1\right) \Delta^{k} \ln p_{g c t}+\varepsilon_{g c t}^{k} \\
\Delta^{k} \ln p_{g c t}=\frac{\omega_{g}}{1+\omega_{g}} \Delta^{k} \ln s_{g c t}+\delta_{g c t}^{k}
\end{gathered}
$$

Donde $\Delta^{k} \ln y_{g c t}=\Delta y_{g c t}-\Delta y_{g k t}, \varepsilon_{g c t}^{k}=\varepsilon_{g c t}-\varepsilon_{g k t} \mathrm{y} \delta_{g c t}^{k}=\delta_{g c t}-\delta_{g k t}$. 
A continuación, multiplicamos las dos ecuaciones entre sí, y tomamos la media de sus componentes a lo largo del tiempo con el fin de obtener la ecuación que estimaremos:

$$
\bar{Y}_{g c}=\theta_{1} \bar{X}_{1 g c}+\theta_{2} \bar{X}_{2 g c}+\bar{u}_{g c}
$$

Donde la barra superior indica que son valores medios tomados a lo largo del tiempo y donde

$$
Y_{g c t}=\left(\Delta^{k} \ln p_{g c t}\right)^{2}, X_{1 g c t}=\left(\Delta^{k} \ln s_{g c t}\right)^{2}, X_{1 g c t}=\left(\Delta^{k} \ln p_{g c t} \Delta^{k} \ln s_{g c t}\right) \mathrm{y} u_{g c t}\left(\varepsilon_{g c t}^{k} \delta_{g c t}^{k}\right)
$$

La estrategia de identificación consiste en asumir que los términos de error de las ecuaciones de demanda y de oferta para cada variedad no están correlacionadas; esto es, $E\left[\varepsilon_{g c t} \delta_{g c t}\right]=0$ (en otros términos, $\bar{u}_{g c} \rightarrow 0$ en el límite de probabilidad cuando $T \rightarrow \infty)$. Esto implica que el término de error no está correlacionado con ninguna variable de la parte derecha de la ecuación [10] y podemos explotar esos momentos en una estimación por variables instrumentales (VI) en la ecuación [10]. Feenstra (1994) utiliza la estructura de panel de los datos para controlar por endogeneidad usando los efectos fijos país como variables instrumentales con el fin de obtener estimaciones consistentes de los parámetros $\theta_{1}$ y $\theta_{2}$.

El problema al estimar $\left(\theta_{1}, \theta_{2}\right)$ a través de la ecuación [10] es que no siempre es posible obtener valor con sentido económico para $\sigma_{g}$. Es ese caso, Broda y Weinstein (2006) usan un método de búsqueda de los valores de $\sigma_{g}>1$ que minimicen la función objetivo por el método general de momentos (MGM) equivalente al del método de estimación por VI.

Debemos comentar tres aspectos econométricos en relación a la estimación de la ecuación [10]. Primero, como hemos diferenciado respecto a un país de referencia $\mathrm{k}$, cada bien necesita tener al menos una variedad (un país) que siempre esté presente en el todo periodo, sin interrupción; esto puede afectar al número de años disponibles en el cálculo. Segundo, el uso de valores unitarios en lugar de precios es inherente a los datos de importaciones. Como los precios están sujetos a errores de medida, ello afectara al tamaño de la varianza muestral. Feenstra (1994) propone incluir una constante en la ecuación [10] para atenuar los posibles efectos asociados a errores de medida. Tercero, para ganar eficiencia en la estimación podemos emplear el método de VI ponderado. Broda y Weinstein (2006) demuestran que la varianza muestral se reduce a medida que aumenta la cantidad física importada de cada variedad así como el número de años seleccionada (el cual puede verse afectado por la selección de país de referencia k) ${ }^{2}$.

2 Siguiendo a BRODA y WEINSTEIN (2006) calculamos la varianza muestral como $\left(1 / T^{3}\right)\left(\left(1 / q_{g c t}\right)+\left(1 / q_{g c t-1}\right)\right)$, donde $\mathrm{T}$ es el número de años y q es la cantidad física de variedad (medido en kilogramos). 


\subsection{Limitaciones de esta metodología}

La metodología descrita se usa para calcular la ganancia de bienestar derivada del consumo de nuevas variedades importadas. Pero ello se realiza bajo un supuesto crítico: no hay competencia entre las variedades domésticas y las variedades importadas (en parte justificado por el uso de preferencias CES). En el caso extremo de que el número de variedades domésticas fuera constante, la competencia entre variedades domésticas e importadas no sería importante. Sin embargo, es razonable esperar que la competencia extranjera tendrá cierto impacto sobre las variedades domésticas, bien reduciendo su número, bien ajustando los márgenes de beneficios. En ambos casos las ganancias de bienestar totales estarían sobreestimadas porque parte de las ganancias de bienestar por acceso a nuevas variedades importadas se vería compensado en parte por la pérdida de bienestar, bien por la reducción en el número de variedades domésticas o por la caída en los márgenes empresariales ${ }^{3}$.

Dos trabajos recientes investigan el sesgo potencial resultante de ignorar los efectos de una sustitución entre variedades domésticas e importadas. Feenstra y Weinstein (2010) usan preferencias translog para evaluar conjuntamente cambios en el número de nuevas variedades importadas y cambios en los márgenes empresariales sobre las ganancias de bienestar en los EEUU. Sus resultados revelan que las ganancias totales son del mismo tamaño que las estimadas por Broda y Weinstein (2006) utilizando preferencias CES, pero que la composición de las ganancias es diferente: el cambio en los márgenes empresariales representa el 33 por 100 de la ganancia total y el cambio en el número de variedades importadas representa un 66 por 100. Sin embargo, es difícil hacer comparaciones entre estos dos trabajos sobre el impacto mayor o menor de la expansión de nuevas variedades importadas debido a que usan enfoques diferentes.

Ardelean y Lugovsky (2010) encuentran que la sustitución entre variedades domésticas e importadas es relevante a nivel sectorial: en algunos sectores manufactureros de EEUU el cambio total en el número de variedades se queda corto mientras que en otros sectores se sobreestima. Sin embargo, en el agregado de las manufacturas, la sustitución entre variedades domésticas e importadas no afecta mucho a las ganancias de bienestar por la importación de nuevas variedades. Este resultado sugiere que estimaciones que usan sólo datos de importaciones no tienden a sobreestimar las ganancias de bienestar a pesar de no tener en cuenta el efecto de la competencia exterior sobre las variedades domésticas.

${ }^{3}$ MELITZ (2003) demuestra que las empresas menos eficientes son «expulsadas» por las empresas extranjeras más productivas, reduciendo el número de variedades domésticas producidas. 


\section{Datos}

En 1988 la cuota de importaciones españolas como porcentaje del PIB era del 19 por 100 y 20 años después del 28 por 100. Junto con el aumento del volumen importador también ha aumentado el número de productos y de variedades importadas. Tanto el número de productos como el número de variedades está determinado por la clasificación de productos utilizada y por la definición de «socio comercial». Nuevos productos aparecen normalmente dentro de categorías de productos que ya existían, lo que conduce a subestimar el crecimiento de las variedades dentro de cada producto, en tanto que el número de productos esté acotado. En este trabajo definimos un producto a nivel de 6 dígitos del Sistema Armonizado y una variedad como la importación de un producto desde un país concreto. La base de datos utilizada son los flujos por países de importaciones anuales de España, en valor y en peso, que publica AEAT-Aduanas desde 1988, a nivel de producto (6 dígitos del sistema armonizado) y de país exportador ${ }^{4}$.

Debido a los cambios que sufre la clasificación del Sistema Armonizado en 1996 y 2002 la definición de producto cambia en el tiempo. Este problema se puede corregir utilizando las Tablas de Transposición que publica Eurostat con el título Update CN Tables, haciendo posible definir códigos de producto que permanecen constantes en el periodo analizado (1988-2006). Por lo tanto, nuestra medición de crecimiento de variedades es muy conservadora pues se restringe a cambios en el número de países exportadores dentro de cada producto. Por lo tanto nuestros resultados ofrecen una medida a la baja de la verdadera ganancia de bienestar asociada al crecimiento de variedades importadas en España ${ }^{5}$.

El Cuadro 1 presenta una descripción básica de la base de datos. El Panel A presenta los datos originales en el primer y último año de la muestra usando los códigos del Sistema Armonizado a 6 dígitos de ese año, 1988 y 2006. El Panel B repite el análisis usando las Tablas de Concordancia de Eurostat, que garantizan que el número de códigos del Sistema Armonizado se mantiene constante durante el periodo de análisis. En 1988 el número de variedades importadas era de 62.509 (4.535 productos y una media de 14 países por producto) y en 2006 había 106.238 (4.535 productos con una media de 23 países). Es evidente que el número de países oferentes por producto casi se ha doblado, evidencia clara de que el número de varie-

${ }^{4}$ Cuando un país se fragmenta después de 1988 se agregan los flujos de los nuevos países (Unión Soviética, República Checa, Yugoslavia). Lo mismo hacemos antes de la reunificación de países (Alemania).

5 Los códigos de Sistema Armonizado se actualizan todos los años pero en algunos años (como 1996, 2002 y 2007) los cambios son mayores que en el resto. Para el caso de España, sin ajuste de códigos en el periodo 1995-1996, la cuota de importaciones de 1995 asociado a productos que entraron y que salieron fue del 14 y 16 por 100 del valor de las importaciones totales de 1995, respectivamente. Después de utilizar la concordancia, las cuotas en 1995 fueron solo de 0,2 y 0,3 por 100, respectivamente. Para EEUU, PIERCE y SCHOTT (2010) demuestran que el uso de las tablas de concordancia es necesario para poder identificar adecuadamente el margen extensivo en el crecimiento de las exportaciones de EEUU, esto es, el valor de las importaciones asociado a la entrada y salida de productos. 
dades ha aumentado. La explicación más general es que el proceso de globalización está contribuyendo a ello, junto con el hecho de que muchos productos son percibidos como diferentes simplemente porque vienen de otros países. Por ejemplo, la reducción en los costes de transporte ha facilitado el acceso de nuevas variedades de producto desde países diferentes. Alternativamente, grandes economías emergentes como China o India son capaces de producir más y más variedades que son importadoras desde los países desarrollados. Pero, como ya hemos explicado, si esos productos son percibidos como diferenciados por el país importador, entonces deberíamos de esperar una ganancia de bienestar por el acceso a más variedades, que es lo que vamos a calcular en la siguiente sección.

\section{CUADRO 1}

DESCRIPCIÓN DE LA BASE DE DATOS

\begin{tabular}{|c|c|c|c|c|c|c|}
\hline Año & Productos & $\begin{array}{c}\text { Número } \\
\text { de códigos } \\
\text { (productos) }\end{array}$ & $\begin{array}{c}\text { Número } \\
\text { medio } \\
\text { (mediana) } \\
\text { de países } \\
\text { exportadores }\end{array}$ & $\begin{array}{c}\text { Número } \\
\text { medio } \\
\text { (promedio) } \\
\text { de países } \\
\text { exportadores }\end{array}$ & $\begin{array}{l}\text { Número } \\
\text { total de } \\
\text { variedades } \\
\text { (producto- } \\
\text { país) }\end{array}$ & $\begin{array}{l}\text { Cuota } \\
\text { sobre im- } \\
\text { portaciones } \\
\text { totales } \\
(\%)\end{array}$ \\
\hline \multicolumn{7}{|c|}{ PANEL A: Sistema Armonizado de 6 dígitos } \\
\hline 1988 & $\begin{array}{l}\text { Todos } \\
\text { En común } \\
\text { No en } 2006 \\
\text { Todos } \\
\text { En común } \\
\text { No en } 1988\end{array}$ & $\begin{array}{r}4.983 \\
4.501 \\
482 \\
5.182 \\
4.501 \\
681\end{array}$ & $\begin{array}{r}11 \\
12 \\
9 \\
17 \\
18 \\
12\end{array}$ & $\begin{array}{l}13,2 \\
13,4 \\
11,4 \\
22,8 \\
22,8 \\
16,5\end{array}$ & $\begin{array}{r}65.952 \\
60.448 \\
5.504 \\
113.904 \\
102.695 \\
11.209\end{array}$ & $\begin{array}{r}100 \\
88 \\
12 \\
100 \\
87 \\
13\end{array}$ \\
\hline \multicolumn{7}{|c|}{ PANEL B: Concordancia códigos Sistema Armonizado 1988-2006 } \\
\hline $\begin{array}{l}1988 \\
2006\end{array}$ & $\begin{array}{l}\text { En común } \\
\text { En común }\end{array}$ & $\begin{array}{l}4.535 \\
4.535\end{array}$ & $\begin{array}{l}12 \\
19\end{array}$ & $\begin{array}{l}13,8 \\
23,4\end{array}$ & $\begin{array}{r}62.509 \\
106.238\end{array}$ & $\begin{array}{l}99,9 \\
99,9\end{array}$ \\
\hline
\end{tabular}

Se puede obtener una mejor idea de qué fuerzas conducen al aumento del número de variedades si se analiza la información a través de los países exportadores. El Cuadro 2 presenta el número de productos exportados a España por país. La primera columna los ordena de mayor a menor en 1988, y las dos siguientes los ordena en 1997 y 2006. No sorprende el ver que los países que más variedades exportan a España son países de tamaño económico grande, de renta per cápita alta, o cercanos geográficamente. Al mirar los cambios en el ranking a lo largo del tiempo surgen algunos resultados interesantes. Primero, todos los países menos dos, Suiza y Japón, han aumentado el número de productos exportados a España. Segundo, tres países, China, India y Turquía, han mejorado posiciones rápidamente en el ranking: China 
se ha movido de la posición 15 a la 8; India ha pasado de la 23 a la 15; Turquía ha pasado de la 35 a la 16. Esto es un claro reflejo del fenómeno de la globalización durante las dos últimas décadas. Tercero, estos tres países son los que más han visto crecer el número de productos exportados a España durante el periodo. Así, por ejemplo, el número de productos exportados por Turquía a España se ha multiplicado por 5, y en el caso de China casi por 3.

CUADRO 2

RANKING DE PAÍSES ORDENADOS POR NÚMERO DE PRODUCTOS IMPORTADOS EN ESPAÑA

\begin{tabular}{|c|c|c|c|c|c|}
\hline & \multicolumn{3}{|c|}{ Ranking en año } & \multirow{2}{*}{$\begin{array}{c}\text { Ratio } \\
\text { de productos } \\
2006 / 1988\end{array}$} & \multirow{2}{*}{$\begin{array}{l}\text { Contribución } \\
\text { al crecimientc } \\
\text { 1988-2006 } \\
\text { (\%) }\end{array}$} \\
\hline & 1988 & 1997 & 2006 & & \\
\hline Francia & 1 & 1 & 2 & 1,04 & 0,4 \\
\hline Alemania & 2 & 2 & 1 & 1,07 & 0,6 \\
\hline Italia & 3 & 3 & 3 & 1,14 & 1,2 \\
\hline Reino Unido & 4 & 4 & 4 & 1,06 & 0,5 \\
\hline Estados Unidos & 5 & 5 & 9 & 1,06 & 0,4 \\
\hline Holanda & 6 & 6 & 5 & 1,19 & 1,3 \\
\hline Bélgica-Luxemburgo & 7 & 7 & 6 & 1,18 & 1,2 \\
\hline Suiza & 8 & 9 & 10 & 0,99 & $-0,2$ \\
\hline Portugal & 9 & 8 & 7 & 1,55 & 2,9 \\
\hline Japón & 10 & 11 & 13 & 0,98 & $-0,1$ \\
\hline Suecia & 11 & 14 & 14 & 1,14 & 0,6 \\
\hline Dinamarca & 12 & 12 & 12 & 1,24 & 1,0 \\
\hline Austria & 13 & 13 & 11 & 1,32 & 1,3 \\
\hline Taiwan & 14 & 15 & 17 & 1,26 & 0,9 \\
\hline China & 15 & 10 & 8 & 2,81 & 4,9 \\
\hline República de Corea & 16 & 16 & 18 & 1,60 & 1,6 \\
\hline Hong Kong & 17 & 21 & 25 & 1,49 & 1,1 \\
\hline Finlandia & 18 & 27 & 30 & 1,16 & 0,4 \\
\hline Canadá & 19 & 19 & 20 & 1,72 & 1,6 \\
\hline Noruega & 20 & 22 & 29 & 1,28 & 0,6 \\
\hline Irlanda & 21 & 18 & 27 & 1,42 & 0,9 \\
\hline Brasil & 22 & 26 & 19 & 1,98 & 2,0 \\
\hline India & 23 & 17 & 15 & 2,91 & 3,3 \\
\hline Israel & 24 & 28 & 28 & 1,82 & 1,3 \\
\hline Mexico & 25 & 20 & 21 & 2,48 & 2,2 \\
\hline Turquía & 35 & 24 & 16 & 5,01 & 3,6 \\
\hline
\end{tabular}

NOTA: En el periodo 1988-1990 hemos considerado como un único país la RDA y la RFA; también hemos tratado como un único país a Bélgica y Luxemburgo en el periodo 1996-2006. 


\section{Resultados}

\subsection{Estimación de las elasticidades de substitución.}

El Cuadro 3 presenta una descripción de los valores estimados de la elasticidad de substitución de cada uno de los productos importados (4.535 según el Sistema Armonizado de 6 dígitos y 2.818 según CUCI a 3 dígitos) durante el periodo 19882006. El valor del percentil 50 (mediana) de la elasticidad es 4,4 y 3,8 , respectivamente; estos valores son similares a los que reporta Broda et al. (2006) para España utilizando el Sistema Armonizado a 3 dígitos. Además, tal y como revelan los resultados, la elasticidad de substitución tiende a ser mayor cuando se utiliza una clasificación de productos más desagregada. La razón es que una definición de «producto» muy genérica (electrodomésticos) incluye sub-productos que los consumidores perciben como muy diferentes (secador de pelo y lavadora de ropa), haciendo que la elasticidad de substitución tienda a ser pequeña.

\section{CUADRO 3}

ESTIMACIÓN DE LAS ELASTICIDADES DE SUBSTITUCIÓN DE PRODUCTOS IMPORTADOS

\begin{tabular}{|l|c|c|c|c|c|c|c|}
\hline & $\begin{array}{c}\text { Núm. } \\
\text { observac. }\end{array}$ & Media & Mediana & pet 5 & pet 95 & Mínimo & Máximo \\
\hline SA 6 dígitos & 4.535 & 6,68 & 4,43 & 2,02 & 14,53 & 1,27 & 199,75 \\
CUCI 3 dígitos & 2.818 & 6,10 & 3,86 & 2,02 & 13,32 & 1,16 & 182,22 \\
\hline
\end{tabular}

\subsection{La ratio de lambdas}

El Cuadro 4 presenta una descripción de la ratio de lambdas para los 4.535 productos analizados del sistema armonizado a 6 dígitos, tal y como aparece definida en la ecuación [4]. Estos valores ilustran el crecimiento o disminución en el número de variedades. Para el periodo entero la mediana de la ratio de lambdas es inferior a la unidad, lo que indica que un sector típico experimentó un aumento en el número de variedades. El valor de 0,92 dice que cualquier categoría de producto en promedio experimentó un crecimiento en el gasto en nuevas variedades del 8 por 100. A modo de comparación podemos utilizar un indicador menos sofisticado basado en contar variedades que aparecen o desaparecen. El dato de recuento (que llamamos «ratio $V »)$ es mucho más pequeño $(0,6<0,92)$, da claramente a entender que hay un gran número de nuevas variedades con cuotas de mercado muy pequeñas. 
CUADRO 4

RATIO DE LAMBDA Y RATIO V

\begin{tabular}{|l|c|c|c|}
\hline & $\begin{array}{c}\text { Ratio de lambda } \\
\text { mediana }\end{array}$ & $\begin{array}{c}\text { [Percentil 5, } \\
\text { Percentil 95] }\end{array}$ & Ratio V \\
\hline Periodo 1988-2006 & 0,926 & {$[0,237,1,601]$} & 0,600 \\
Periodo1988-1997 & 0,982 & {$[0,425,1,598]$} & 0,800 \\
Periodo 1997-2006 & 0,975 & {$[0,479,1,365]$} & 0,765 \\
\hline
\end{tabular}

\subsection{End-point ratio, sesgo de importaciones y ganancias de bienestar}

Las elasticidades y las ratio de lambdas de los Cuadros 3 y 4 sirven para calcular el índice de precios de importación exacto producto por producto (ecuación [3]). Agregando esos índices se obtiene el índice de precios de importación exacto agregado y dividiendo este índice por el índice de precios de importación convencional obtenemos el end-point-ratio o EPR (ecuación [5]).El resultado aparece en la columna 1 del Cuadro 5. Si la ratio es menor que 1, entonces el cambio en variedades ha contribuido a reducir el índice de precios de importación convencional. La columna 2 contiene el sesgo al alza (signo positivo) o a la baja (signo negativo) en el índice de precios de importación convencional. La columna 3 muestra la cuota de las importaciones como porcentaje del PIB en promedio durante el periodo analizado. Al ponderar la inversa de la columna 1 con la cuota de importaciones de la columna 3 se obtiene las ganancias de variedad (GV) como fracción del PIB en la columna 4. Como ejemplo, la primera fila del Cuadro 5 muestra un EPR de 0,951 a lo largo del periodo 1988-2006. Esto representa un sesgo al alza del índice de precios de importaciones convencional de un 4,9 por 100 en el conjunto del periodo. Ponderando el sesgo por la cuota de importaciones del 24 por 100 obtenemos una ganancia de variedad del 1,21 por 100 del PIB de 2006. Esta ganancia puede interpretarse de la siguiente forma: los consumidores españoles están dispuestos a pagar el 1,21 por 100 del PIB en el año 2006 por acceder al mayor número de variedades importadas que había en 2006 comparado con el número de variedades que había en 1988. 


\section{CUADRO 5}

EPR; SESGO DE IMPORTACIONES Y GANANCIAS POR VARIEDADES (GV)

\begin{tabular}{|c|c|c|c|c|}
\hline & $\begin{array}{l}\text { End-point } \\
\text { ratio }\end{array}$ & $\begin{array}{c}\text { Sesgo de } \\
\text { importación }\end{array}$ & $\begin{array}{c}\text { Cuota de } \\
\text { importaciones }\end{array}$ & $\begin{array}{c}\text { Ganancias de } \\
\text { variedad (\% GDP) }\end{array}$ \\
\hline $1988-2006$ & $\begin{array}{c}0,951 \\
{[0,929,0,957]}\end{array}$ & 4,90 & 0,24 & $\begin{array}{c}1,21 \\
{[1,04,1,66]}\end{array}$ \\
\hline 1988-1997 & $\begin{array}{c}0,985 \\
{[0,981,0,988]}\end{array}$ & 1,50 & 0,22 & $\begin{array}{c}0,33 \\
{[0,25,0,40]}\end{array}$ \\
\hline $1997-2006$ & $\begin{array}{c}0,974 \\
{[0,964,0,978]}\end{array}$ & 2,60 & 0,27 & $\begin{array}{c}0,71 \\
{[0,58,0,98]}\end{array}$ \\
\hline
\end{tabular}

NOTA: Los intérvalos de confianza (en paréntesis) se obtienen mediante el método de bootstrapping usando 50 repeticiones.

\subsection{Ganancias de bienestar por origen geográfico}

Hasta el momento hemos calculado la ganancia de bienestar por importación de nuevas variedades sin tener en cuenta su procedencia. Ahora queremos calcular la cuota que representa cada país exportador en dicha ganancia total. Para ello calculamos la contribución de cada país a cada producto, y las sumamos para todos los productos con el fin de obtener la contribución individual de un país exportador. Primero calculamos los ponderadores de cada país en cada producto a partir de la cuota de gasto al final del periodo analizado. La cuota de gasto del «país de interés» se calcula como:

$$
s_{g, r e f, t}=\frac{\sum_{r e f \in I_{g}} p_{g, r e f, t} q_{g, r e f, t}}{\sum_{c \in I_{g}} p_{g c, t} q_{g c, t}}
$$

Donde $I_{g}$ es el conjunto de variedades en común al principio y final del periodo, el subíndice ref indica el conjunto de variedades del país de interés y el subíndice $t$ se refiere en este caso al año 2006. El peso para cada país de interés es simplemente su cuota de gasto dividida por la suma de las cuotas de gasto de los demás países. La ratio de lambda de cada producto de la ecuación [7] se eleva a este peso específico de país exportador para obtener la contribución de cada país al end-point-ratio (EPR) y las ganancias totales por variedades importadas (GV).

El Cuadro 6 muestra la distribución geográfica por grandes áreas de las ganancias de variedades importadas. El continente con la mayor contribución es Asia (35 por 100), seguida de Europa Central y Oriental (17,4 por 100), Europa Occidental (14,6 por 100) y África (14 por 100). 


\section{CUADRO 6 \\ CONTRIBUCIÓN DE LAS ÁREAS \\ GEOGRÁFICAS A LAS GANANCIAS DE VARIEDAD EN ESPAÑA. PERIODO 1988-2006}

\begin{tabular}{|l|c|}
\hline \multicolumn{1}{|c|}{ Grupos de países } & \% de ganancia \\
\hline Europa Occidental & 14,6 \\
Resto de Europa & 17,3 \\
África & 14,3 \\
Asia & 35,2 \\
Latinoamérica & 11,1 \\
Resto de América & 1,8 \\
Antigua Unión Soviética & 5,5 \\
\hline
\end{tabular}

El Cuadro 7 muestra la contribución de una selección de países a las ganancias totales de bienestar por importación de nuevas variedades desde esos países a lo largo del periodo 1988-2006. China es el país que tiene el mayor peso con un 11,8 por 100 de la ganancia total (equivalente al 0,14 por 100 del PIB de España en 2006). La contribución de China es enorme si tenemos en cuenta que sus exportaciones a España sólo representaban un 5 por 100 del total de importaciones españolas entre 1988 y 2006. Otras seis economías emergentes, Indonesia, Egipto, Turquía, Brasil, India y Rusia, representan en conjunto el 19 por 100 del total de ganancias. Los países de la Europa Central también realizan una contribución significativa: República Checa y Eslovaquia, Polonia, Hungría representan el 11 por 100 de las ganancias totales por nuevas variedades. Por último tres países de la UE, Portugal, Holanda e Irlanda, contribuyen cada uno con un 2 por 100. Los cuatro mayores países exportadores de la UE contribuyen con un 3,8 por 100 en total ( 1 por 100 de Francia, y 0,7 por 100 de Reino Unido, Alemania e Italia). El conjunto de la UE-15 representa el 13,6 por 100 de las ganancias totales por acceso a un número mayor de variedades importadas. 
CUADRO 7

CONTRIBUCIÓN DE LOS PAÍSES EXPORTADORES A LAS GANANCIAS DE VARIEDAD EN ESPAÑA EN EL PERIODO 1988-2006

\begin{tabular}{|l|c|l|c|l|l|}
\hline \multicolumn{1}{|c|}{ País } & $\begin{array}{c}\text { \% de las } \\
\text { ganancias }\end{array}$ & \multicolumn{1}{|c|}{ País } & $\begin{array}{c}\text { \% de las } \\
\text { ganancias }\end{array}$ & \multicolumn{1}{c|}{ País } & $\begin{array}{c}\text { \% de las } \\
\text { ganancias }\end{array}$ \\
\hline Portugal & 2,4 & China & 11,8 & Rep. Checa & 4,4 \\
Holanda & 2,3 & Indonesia & 4,4 & Polonia & 3,2 \\
Irlanda & 2,2 & Egipto & 4,2 & Hungría & 3,2 \\
Francia & 1,0 & Turquía & 3,5 & Rumania & 1,2 \\
Bélgica-Lux. & 0,9 & Brasil & 2,6 & Bulgaria & 0,9 \\
Austria & 0,9 & India & 2,3 & Eslovenia & 0,7 \\
Reino Unido & 0,7 & Rusia & 2,2 & & \\
Alemania & 0,7 & Marruecos & 1,4 & & \\
Italia & 0,7 & & & & \\
Finlandia & 0,5 & & & & \\
Suecia & 0,5 & & & & \\
Dinamarca & 0,4 & & $\mathbf{1 8 , 9}$ & PECO & \\
Grecia & 0,4 & & BRIC & & \\
\hline UE-15 & $\mathbf{1 3 , 6}$ & & & & \\
\hline
\end{tabular}

\section{Conclusiones}

Una de las consecuencias de la globalización es la expansión del número de variedades importadas desde muy diversos países. En este trabajo hemos aplicado la metodología propuesta por Feenstra (1994) y mejorada por Broda y Weinstein (2006) para investigar las ganancias de bienestar por el crecimiento de variedades importadas en España desde 1988 hasta 2006. El trabajo estima que el efecto de las nuevas variedades importadas entre 1988 y 2006 en el aumento del bienestar es equivalente al 1,2 por 100 del PIB de 2006, lo que consideramos es un valor mínimo dado las condiciones restrictivas que impone la metodología implementada.

Por países, China aparece como el país que más ha contribuido al aumento de bienestar por gasto en nuevas variedades (12 por 100). Le siguen Indonesia, Egipto, Turquía, la República Checa, Polonia y Hungría, cada una con más del 3 por 100 de contribución a la ganancia total. Por último, la UE-15 tiene una contribución modesta (13,6 por 100), con Portugal, Holanda e Irlanda contribuyendo con un 2 por 100 cada uno a las ganancias de bienestar.

Otra contribución de este trabajo es la disponibilidad de estimaciones de las elasticidad de substitución para productos importados a partir de distintas clasificaciones como el sistema armonizado a 6 dígitos o CUCI a 3 dígitos (esta base de datos así como los programas para su construcción están disponibles para quien lo solicite a los autores). 


\section{Referencias bibliográficas}

[1] ARDELEAN, A. y LUGOVSKY, V. (2010): «Domestic Productivity and Variety Gains from Trade», Journal of International Economics, 80, 280-291.

[2] ARMINGTON, P. (1969): "A Theory of Demand for Products Distinguished by Place of Production», International Monetary Fund Staff Papers, 16, 159-178.

[3] BLONIGEN, B. y SODERBERY, A. (2010): «Measuring The Benefits Of Product Variety With An Accurate Variety Set», Journal of International Economics, 82, 168180.

[4] BRODA, C. y WEINSTEIN, D. E. (2006): «Globalization and the Gains from Variety», Quarterly Journal of Economics, 121, 541-585.

[5] BRODA, C.; GREENFIELD, J. y WEINSTEIN, D. (2006): «From Groundnuts to Globalization: A Structural Estimate of Trade and Growth», NBER Working Paper No. 12512.

[6] FEENSTRA, R. C. (1994): «New Product Varieties and the Measurement of International Prices», American Economic Review, 84, 157-177.

[7] FEENSTRA, R. C. (1994), y WEINSTEIN, D. E. (2010): «Globalization, Markups and the US price level», NBER Working Papers 15749.

[8] KLENOW, P. J. y RODRIGUEZ-CLARE, A. (1997): «Quantifying Variety Gains from Trade Liberalisation», Working Paper, University of Chicago.

[9] KRUGMAN, P. (1979): «Increasing Returns, Monopolistic Competition, and International Trade», Journal of International Economics, 9, 469.479.

[10] KRUGMAN, P. (1980): «Scale Economies, Product Differentiation, and the Pattern of Trade», American Economic Review, LXX, 950.959.

[11] MELITZ, M. J. (2003): «The Impact of Trade on Intra-Industry Reallocations and Aggregate Industry Productivity», Econometrica, 71(6), 1695-1725.

[12] MOHLER, L. (2009): «Globalization and the Gains from Variety: The Case of a Small Open Economy», FIW - Working Paper 31 (April)

[13] MOHLER, L. y SEITZ, M. (2010): «The Gains from Variety in the European Union», Munich Discussion Paper N. 2010-24.

[14] PIERCE, J. y SCHOTT, P. (2010): Concording U.S. Harmonized System Codes Over Time, mimeo (http://www.som.yale.edu/faculty/pks4/sub_international.htm).

[15] ROMER, P. M. (1994): «New Goods, Old Theory, and the Welfare Costs of Trade Restrictions», Journal of Development Economics, 43, 5-38.

[16] SATO, K. (1976): «The Ideal Log-Change Index Number», Review of Economics and Statistics, 58, 223-228. 


\section{APÉNDICE}

Fórmulas de los ponderadores para el cálculo de la ratio de lambdas, EPR y GV

$\omega_{\text {gct }}$ son las ponderaciones (ideal log-change weights) a nivel de variedad,

$$
\omega_{g c t}=\frac{\left(s_{g c t}-s_{g c t-1}\right)\left(\ln s_{g c t}-\ln s_{g c t-1}\right)}{\left.\sum_{c \in I_{g}}\left(s_{g c t}-s_{g c t-1}\right)\left(\ln s_{g c t}-\ln s_{g c t-1}\right)\right)}
$$

esto es, la media harmónica delas participaciones en el coste total de cada variedad:

$$
s_{g c t}=\frac{p_{g c r} q_{g c r}}{\sum_{c \in I_{g}} p_{g c r} q_{g c r}} \text { para } r=t, t-1
$$

donde $I_{g}$ es el conjunto de variedades que están disponibles al principio y final del periodo, y $p_{g c r} q_{g c r}$ es el valor de la importación de la variedad c en el periodo $r$.

$\omega_{\text {gct }}$ son los ponderadores (log-change ideal weights) a nivel de producto:

$$
\omega_{g t}=\frac{\left(s_{g t}-s_{g t-1}\right)\left(\ln s_{g t}-\ln s_{g t-1}\right)}{\left.\sum_{g \in G}\left(s_{g t}-s_{g t-1}\right)\left(\ln s_{g t}-\ln s_{g t-1}\right)\right)}
$$

esto es, la media harmónica de las participaciones en el coste total de cada producto

$$
s_{g r}=\frac{\sum_{c \in I r} p_{g c r} q_{g c r}}{\sum_{g \in G} \sum_{c \in I_{g}} p_{g c t} q_{g c r}} \text { para } r=t, t-1
$$

donde $G$ es el conjunto de productos que están disponibles para el consumo a lo largo de todo el periodo, $I_{g}$ es el conjunto de variedades que están disponibles al principio y final del periodo, y $p_{g c r} q_{g c r}$ es el valor de la importación de la variedad $c$ en el periodo $r$.

$\omega_{t}^{M}$ es la ponderación (log-change ideal weights) a nivel de importaciones agregadas,

$$
\omega_{t}^{M}=\left(s_{M t}-S_{M t-1}\right)\left(\ln s_{M t}-\ln S_{M t-1}\right)
$$


donde

$$
s_{g r}=\frac{\sum_{g \in G} \sum_{c \in I_{g}} p_{g c t} q_{g c t}}{G D P_{t}}
$$

el numerador $s_{M t}$ de es la suma de todas las importaciones de bienes en el año final del periodo $t$ y el denominador es el PIB, ambos en precios corrientes. 